\title{
Drtopedia de control del daño infantil en zona de combate
}

\author{
Tamburri Bariain R. ${ }^{1 a}$, Navarro Suay R..$^{2 a}$, Porter M. ${ }^{3 b}$, Bodega Quiroga I. ${ }^{4 a}$, Sáenz Casco L. 5a
}

Sanid. mil. 2015; 71 (2): 95-97; ISSN: 1887-8571

\section{RESUMEN}

El objeto de esta comunicación breve es presentar un caso de fractura pediátrica tratada mediante premisas de Ortopedia de Control del Daño en el segundo escalón quirúrgico español de Camp Arena, Herat, Afganistán.

PALABRAS CLAVE: Blast, Fractura pediátrica, Ortopedia de control del daño, ROLE 2.

\section{Pediatrics Damage Control Ortopaedic Surgery in Area of Operations}

SUMMARY: This is case report about a femoral shaft pediatric fracture treated with Orthopaedic Damage Control Means completed at the Second Surgical Echelon in Herat, Afghanistan. We show the benefits of this kind of procedures.

KEYWORDS: Blast Injury, Pediatric fracture, Damage control surgery, ROLE 2.

\section{INTRODUCCIÓN}

Las fracturas de la diáfisis femoral son fracturas de alta energía y están frecuentemente asociados a traumatismos de tráfico y con menos frecuencia son de origen patológico ${ }^{1,2}$, y en zonas de guerra o en proceso de pacificación al efecto directo de explosivos o su onda de blast. En este tipo de escenarios son los explosivos improvisados o las minas antipersonal los agentes lesionales más frecuentemente implicados ${ }^{3-5}$. Las fracturas de fémur suponen dentro del conjunto de las fracturas infantiles menos del 1\% de todas las fracturas en la edad pediátrica ${ }^{6}$. Estas pueden ocurrir en cualquier segmento de edad, pero es el rango de edad entre los 11 y los 12 años el que engloba el $75 \%$ de los casos pediátricos; Las diferencias de densidad ósea entre la edad infantil y adulta explican que la ratio entre ambas sea de 130:17.

\section{CASO CLÍNICO}

Paciente varón de cinco años de edad que tras sufrir un atentado con explosivo improvisado (Improvised Explosive Device$I E D)$ en un lugar público es evacuado sobre un centro asistencial local siendo diagnosticado en primera instancia de fractura diafisaria de fémur izquierdo. La fractura no es adecuadamente reducida (Figura 1) y se inmoviliza con una férula suropédica. Se evacua sobre el segundo escalón quirúrgico militar español ROLE 2 de Herat, Afganistán para tratamiento definitivo.

${ }^{1}$ Cte. Médico. Servicio de Cirugía Ortopédica y Traumatología

${ }^{2}$ Cte Médico. Servicio de Anestesiología, Reanimación y Terapéutica del dolor.

${ }^{3}$ Lieutenant Colonel Dr. US Army Medical Corps.

${ }^{4}$ Cte Médico. Servicio de Cirugía General.

${ }^{5}$ Cte Médico. Servicio de Medicina Intensiva.

a Hospital Central de la Defensa "Gómez Ulla". Instituto Mixto de Investigación Biosanitaria de la Defensa (IMIDEF). Madrid. España.

b 909th Forward Surgical Team, Chicago, IL. Department of the Army. United States of America.

Dirección para correspondencia: rtambar@oc.mde.es

Recibido: 8 de mayo de 2014

Aceptado: 25 de junio de 2014.
Tras constatarse la inaceptable reducción fractuaria, se decide la realización de un procedimiento quirúrgico de Ortopedia de Control del Daño que consiste en una reducción e inmovilización mediante fijación externa.

En ausencia de varillas endomedulares elásticas tipo TENS o fijadores externos para la edad pediátrica se decide una fijación

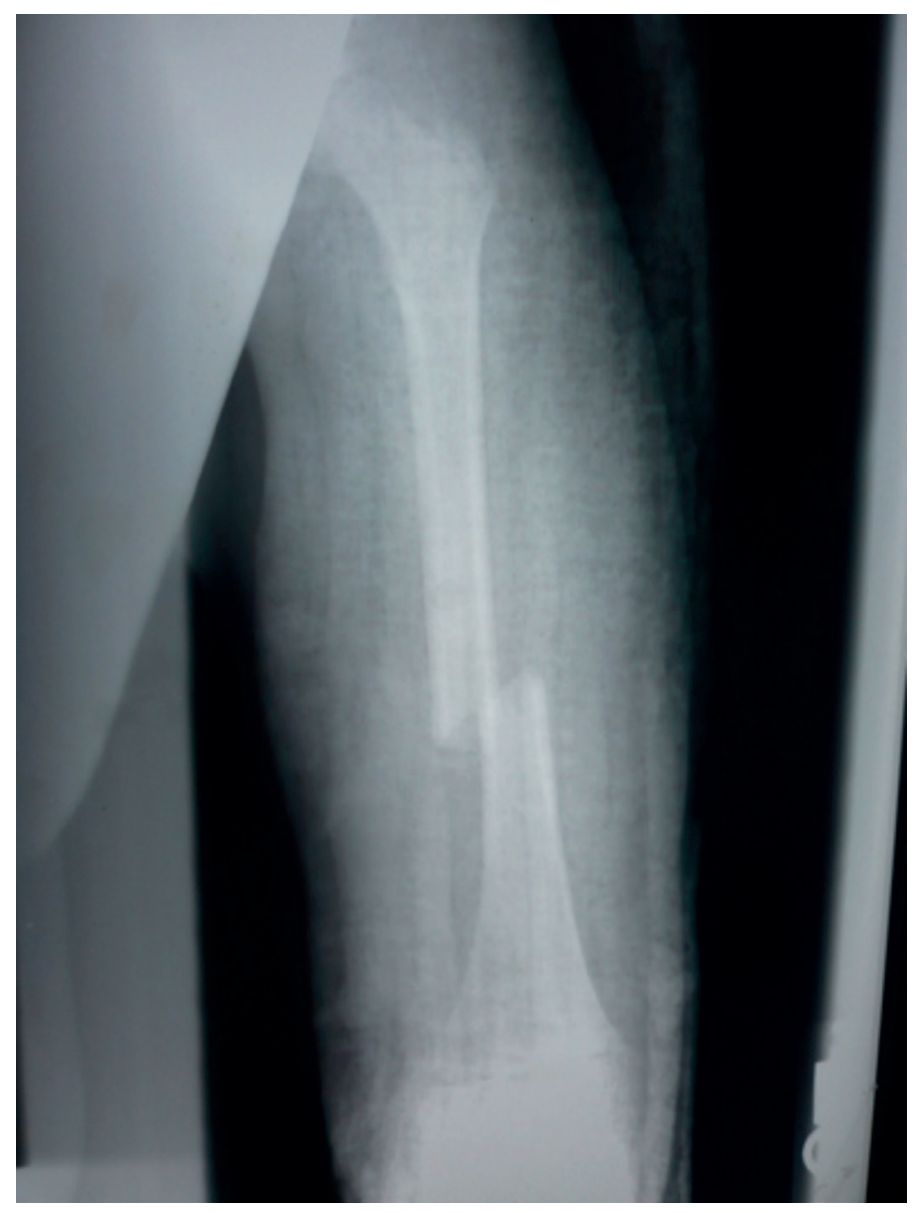

Figura 1. Inaceptable reducción inicial de fractura diafisaria de fémur. 
externa mediante un fijador externo de muñeca (utilizado como método de circunstancias) sobre diáfisis femoral izquierda.

En quirófano y previa optimización anestésica se procede al tiempo quirúrgico que implica un desbridamiento de las partes blandas necróticas con lavado pulsátil profuso. Mediante escopia se confirma la presencia de una fractura de diáfisis femoral de trazo transverso e inestable. Se procede a reducción cerrada con control de escopia de la fractura, realizándose una técnica de fijación externa mediante implantación de un fijador externo Stryker ${ }^{\mathrm{TM}}$ TRIAX (Fijador externo de muñeca y pequeños fragmentos utilizado aquí como método de circunstancias) en configuración monoplano y monotubo. Dos pines proximales y dos pines distales y comprobación bajo escopia de la correcta colocación de los pines (Figura 2).

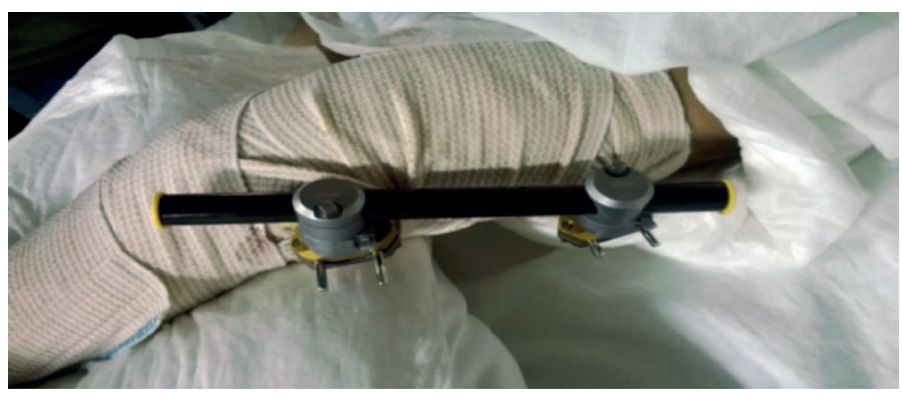

Figura 2. Fijación externa Stryker TRIAX monotubo sobre diáfisis femoral. Inmediato postoperatorio.

\section{RESULTADOS}

Se realizó seguimiento clínico y radiológico de la fractura con curación completa de la lesión de partes blandas. Se consigue callo de fractura progresivo y suficiente con alineación satisfactoria de los extremos fractuarios (Figuras 3 y 4). Tras retirada de fijación externa se consigue la deambulación autónoma, estable e indolora.

\section{DISCUSIÓN}

Las fracturas de diáfisis femoral entre los 5 y los 15 años. Opciones terapeúticas.

\section{La tracción transesquelética}

Todavía es ampliamente usada en países en desarrollo y áreas de conflicto. El procedimiento de tracción transfemoral con estribo es un procedimiento que implica bajo riego quirúrgico. La tracción en exclusiva durante un periodo de entre 6 y 8 semanas tiene como alternativa el uso de la tracción durante 3 a 4 semanas continuada con una inmovilización con yeso cerrado en espica durante 2 o 3 semanas más ${ }^{8,9}$.

Una alternativa muy utilizada en el pasado fue la tracción bilateral de ambos fémures sobre ambos miembros inferiores ferulizados. El tratamiento con la llamada férula de Weber permitía un óptimo control rotacional e implicaba una mayor duración de la tracción que debía seguirse de dos semanas adicionales con yeso cerrado tipo espica ${ }^{10}$.

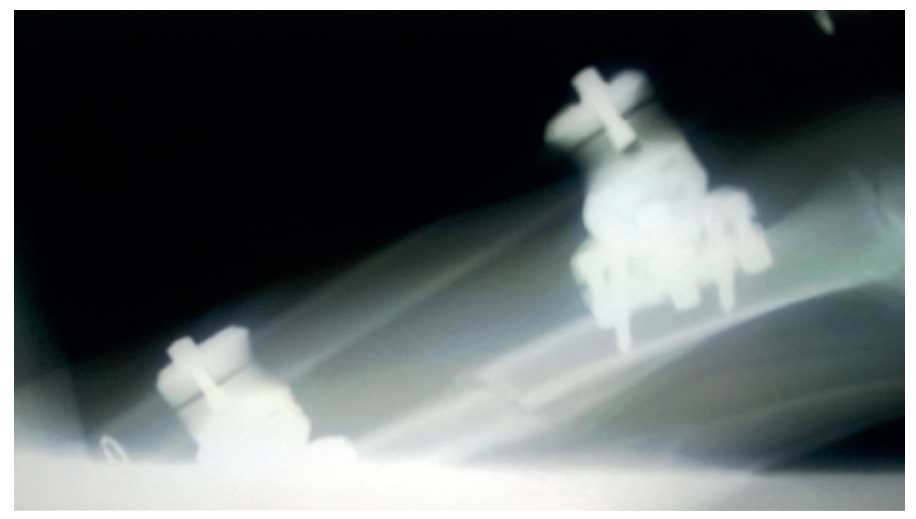

Figura 3. Control radiológico con proyección lateral de la diáfisis del fémur tras procedimiento.

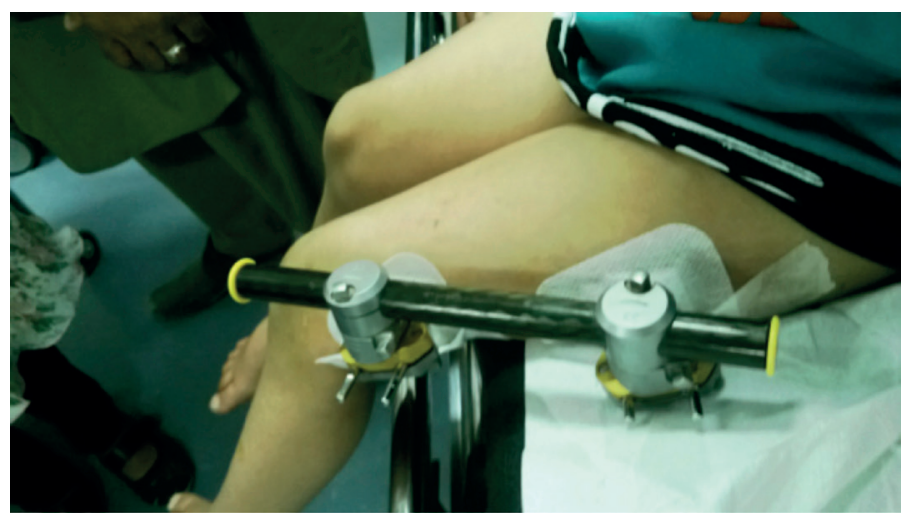

Figura 4. Curación completa de partes blandas.

\section{El yeso en espica}

El uso del llamado yeso en espica es un método de tratamiento de bajo riesgo usado todavía en algunos centros habiéndose utilizado sobre todo en el tramo de edad entre los $7^{11}$ y los 10 años $^{12}$. De cualquier manera, la aplicación del yeso de espica con un correcto ajuste y una correcta aplicación de los principios mecánicos que la rigen no es una cuestión sencilla.

En su aplicación sobre una tabla denominada a su vez tabla de espica, se deben evitar los defectos de consolidación en varo y malrotación. Se aplica una flexion de $45^{\circ}$ sobre cadera y rodilla que puede ser suficiente como maniobra de reducción. El miembro afecto y respecto al plano longitudinal presentará abducción mínima o neutra. En caso de mala reducción puede ser necesaria la realización de ventanas o cuñas sobre el yeso. Las fracturas transversas y oblicuas pueden desarrollar acortamiento ${ }^{13}$. La retirada final del yeso se realiza a las 6-8 semanas con un retorno a la actividad completo a las 3 ó 4 semanas ${ }^{14}$. Un alternativa a este tratamiento pero siguiendo los mismo principios biomecánicos es el uso de ortesis termoconformadas tipo brace ${ }^{15}$ o de la ortesis en espica con apoyo en tres puntos ${ }^{16}$. Ambos métodos evitan la hospitalización prolongada y permiten el tratamiento domiciliario.

\section{Los fijadores externos}

Ha sido un dispositivo de amplio uso como tratamiento de las fracturas de diáfisis femoral durante los últimos 20 años. La 


\section{Ortopedia de control del daño infantil en zona de combate}

fijación externa y estable de la fractura permite una carga precoz del miembro junto a un retorno precoz a la actividad ${ }^{17,18}$.

La mayoría de las complicaciones se han referido en relación con infección de los pines, aunque su tasa de infección suele ser mayor cuando se utilizan los fijadores externos con otros fines (intentos de elongación de miembros o discrepancias de longitud).

En las fracturas transversas de fémur (como es la que nos ocupa en este caso) el fijador debe continuarse en su uso durante tres meses, mientras que en fracturas con trazos espiroideos puede ser suficiente con dos o menos incluso. La refractura después de la retirada del fijador externo puede ocurrir hasta en un $10 \%$ de los casos, implicando por tanto un segundo periodo de morbilidad.

\section{Los dispositivos endomedulares}

Con la introducción de las varillas endomedulares de titanio durante los últimos años, los fijadores externos han perdido su papel terapéutico dominante aunque su uso sigue estando vigente para fracturas abiertas tipo Gustilo 2 y 3 , fracturas consecuencia de la onda de blast asociada a explosivos y heridas por arma de fuego ${ }^{19}$.

El uso de varillas elásticas de titanio endomedulares se ha convertido en la actualidad en el método de elección en las fracturas de diáfisis femoral en edad escolar. Se han desarrollado diferentes tipos de dispositivos elásticos y endomedulares como los franceses denominados clavos de Nancy o ESIN (Elastic Stable Intramedullary Nailing) ${ }^{20-22}$. El sistema se ha extendido a toda Europa ${ }^{23-25}$ y Norteamérica siendo el método de elección para este tipo de fracturas en la actualidad ${ }^{26-28}$.

Otros dispositivos de concepción similar son los llamados TENS (Titanium Endomedullary Nail System) de uso generalizado en la actualidad y que ha sustituido parcialmente al fijador externo como método de fijación en muchos centros ${ }^{29-31}$. En un estudio randomizado de Bar-on et al. se han constatado unos mejores resultados en comparación con los referidos a la fijación externa $^{21}$

\section{BIBLIOGRAFÍA}

1. Landin LA. Fracture patterns in children. Analysis of 8682 fractures with special reference to incidence, etiology and secular changes in Swedish urban population 1950-1979. Acta Orthop Scand Suppl 1983; 202: 1-109.

2. Hedlund R, Lindgren $U$. The incidence of femoral shaft fractures in children and adolescents. J Pediatr Orthop 1986; 6: 47-50.

3. Bumbasirević M, Lesic A, Mitkovic M, Bumbasirević V. Treatment of blast injuries of the extremity. J Am Acad Orthop Surg. 2006;14(10 Spec No.):S77-81.

4. Haidukewych GJ. Temporary external fixation for the management of complex intra- and periarticular fractures of the lower extremity. J Orthop Trauma. 2002 Oct;16(9):678-85.

5. Labeeu F, Pasuch M, Toussaint P, Van Erps S. External fixation in war traumatology: report from the Rwandese war (October 1, 1990 to August 1, 1993). J Trauma. 1996 Mar;40(3 Suppl):S223-7.

6. Cooper C, Dennison EM, Leufkens HG, et al. Epidemiology of childhood fractures in Britain: a study using the general practice research database. Bone Mineral Res 2004; 19: 1976-1981.
7. Kasser JR, Beaty JH. Femoral shaft fractures. In: Beaty JH, Kasser JR, eds. Rockwood and Wilkins' Fractures in Children, 5th ed. Philadelphia: Lippincott-Williams \& Wilkins; 2001: 941-980.

8. Hinton RY, Lincoln A, Crockett MM, et al. Fractures of the femoral shaft in children. Incidence, mechanisms, and sociodemographic risk factors. J Bone Joint Surg 1999; 81: 500-509.

9. Taitz J, Moran K, O’Meara M. Long bone fractures in children under 3 years of age: is abuse being missed in Emergency Department presentations? J Paediatr Child Health 2004; 40: 170-174.

10. Banaszkiewicz PA, Scotland TR, Myerscough EJ. Fractures in children younger than age 1 year: importance of collaboration with child protection services. J Pediatr Orthop 2002; 22: 740-744.

11. Schwendt RM, Werth C, Johnson A. Femur shaft fractures in toddlers and young children: rarely from child abuse. J Pediatr Orthop. 2000 JulAug;20(4):475-81.

12. Thompson JD, Buehler KC, Sponseller PD, et al. Shortening in femoral shaft fractures in children treated with spica cast. Clin Orthop Rel Res 1997; 338 74-78.

13. Hughes BF, Sponseller PD, Thompson JD. Pediatric femur fractures: effects of spica cast treatment on family and community. J Pediatr Orthop 1995; 15 : 457-460.

14. Gross RH, Davidson R, Sullivan JA, et al. Cast brace management of the femoral shaft fracture in children and young adults. J Pediatr Orthop 1983; 3: $572-582$.

15. Guttmann GG, Simon R. Three-point walking spica cast: an alternative to early or immediate casting of femoral shaft fractures in children. J Pediatr Orthop 1988; 8: 699-703.

16. Aronson J, Tursky EA. External fixation of femur fractures in children. J Pediatr Orthop 1992; 12: 157-163.

17. Davis TJ, Topping RE, Blanco JS. External fixation of pediatric femoral fractures. Clin Orthop Relat Res 1995; 318:191-198.

18. Hull JB, Bell MJ. Modern trends for external fixation of fractures in children: a critical review. J Pediatr Orthop B 1997; 6:103-109.

19. Weinberg AM, Hasler CC, Leitner A, et al. External fixation of pediatric femoral shaft fractures. Europ J Trauma 2000; 1:25-32.

20. Hedin H, Hjorth K, Rehberg L. External fixation of displaced femoral shaft fractures in children: a consecutive study of 98 fractures. J Orthop Trauma 2003; 17: 250-256

21. Bar-On E, Sagiv S, Porat S. External fixation or flexible intramedullary nailing for femoral shaft fractures ion children. J Bone Joint Surg 1997; 79 B 975-978.

22. Ramseier LE, Bhaskar AR, Cole WG, et al. Treatment of open femur fracture in children: comparison between external fixator and intramedullary nailing. J Pediatr Orthop 2007; 27:748-750.

23. Metaizeau JP. L'ostéosynthèse chez l'enfant. Embrochage centromédullaire élastique stable. Sauramps, Montpellier 1988:77-84.

24. Lascombe P, Métaizeau JD. Fracture du femur. In: Lascombe P,ed. Embrochage centromédullaire élastique stable. Issy-les-Moulineaux: Elsevier Masson SAS; 2006: 207-240.

25. Dietz HG, Schmittenbecher PP, Illing P. Intramedulläre Osteosynthese am Oberschenkel. In: Intramedulläre Osteosynthese im Wachstumsalter. München: Urban \& Schwarzenberg; 1997: 135-168.

26. Mazda K, Khairouni A, Pennecot GF, et al. Closed flexible intramedullary nailing of the femoral shaft fractures in children. J Pediatr Orthop B 1997; 6: 198-202.

27. Parsch K. Modern trends in internal fixation of femoral shaft fractures in children. A critical review. J Pediatr Orthop Part B 1997; 6: 117-125.

28. Ho CA, Skaggs DL, Tang CW, et al. Use of flexible intramedullary nails in pediatric femur fractures. J Pediatr Orthop 2006; 26: 497-504.

29. Till H, Huettl B, Knorr P, et al. Elastic stable intramedullary nailing (ESIN) provides good long-term results in pediatric long bone fractures. Eur J Pediatr Surg 2000; 10: 319-322.

30. Carey TP, Galpin RD. Flexible intramedullary nail fixation of pediatric femoral fractures. Clin Orthop Relat Res 1996; 332:110-118.

31. Flynn JM, Hresko T, Reynolds RA, et al. Titanium elastic nails for pediatric femur fractures: a multicenter study of early results with analysis of complications. J Pediatr Orthop 2001; 21: 4-8. 